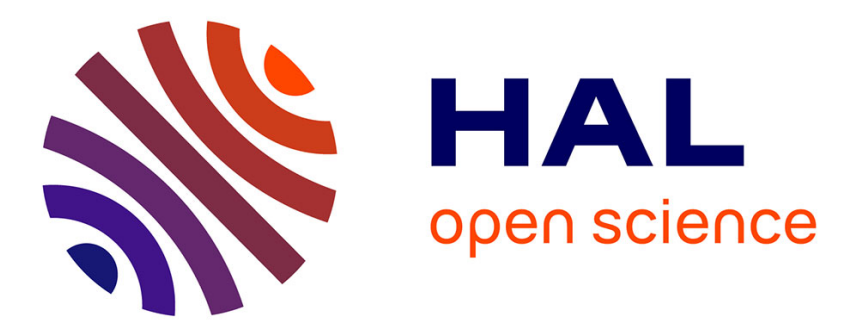

\title{
Fast and Robust Object Segmentation with the Integral Linear Classifier
}

David Aldavert, Arnau Ramisa, Ricardo Toledo, Ramon Lopez de Mantaras

\section{To cite this version:}

David Aldavert, Arnau Ramisa, Ricardo Toledo, Ramon Lopez de Mantaras. Fast and Robust Object Segmentation with the Integral Linear Classifier. CVPR 2010 - 23rd IEEE Conference on Computer Vision \& Pattern Recognition, Jun 2010, San Francisco, United States. pp.1046-1053, 10.1109/CVPR.2010.5540098 . inria-00548642

\section{HAL Id: inria-00548642 \\ https://hal.inria.fr/inria-00548642}

Submitted on 20 Dec 2010

HAL is a multi-disciplinary open access archive for the deposit and dissemination of scientific research documents, whether they are published or not. The documents may come from teaching and research institutions in France or abroad, or from public or private research centers.
L'archive ouverte pluridisciplinaire HAL, est destinée au dépôt et à la diffusion de documents scientifiques de niveau recherche, publiés ou non, émanant des établissements d'enseignement et de recherche français ou étrangers, des laboratoires publics ou privés. 


\section{Fast and Robust Object Segmentation with the Integral Linear Classifier}

\author{
David Aldavert \\ Computer Vision Center \\ Dept. Computer Science \\ Univ. Autònoma de Barcelona \\ Ramon Lopez de Mantaras \\ Artificial Intelligence Research \\ Institute (IIIA-CSIC) \\ Campus UAB
}

\author{
Arnau Ramisa \\ INRIA-Grenoble \\ Artificial Intelligence Research \\ Institute (IIIA-CSIC) \\ Ricardo Toledo \\ Computer Vision Center \\ Dept. Computer Science \\ Univ. Autònoma de Barcelona
}

\begin{abstract}
We propose an efficient method, built on the popular Bag of Features approach, that obtains robust multiclass pixellevel object segmentation of an image in less than $500 \mathrm{~ms}$, with results comparable or better than most state of the art methods. We introduce the Integral Linear Classifier (ILC), that can readily obtain the classification score for any image sub-window with only 6 additions and 1 product by fusing the accumulation and classification steps in a single operation. In order to design a method as efficient as possible, our building blocks are carefully selected from the quickest in the state of the art. More precisely, we evaluate the performance of three popular local descriptors, that can be very efficiently computed using integral images, and two fast quantization methods: the Hierarchical K-Means, and the Extremely Randomized Forest. Finally, we explore the utility of adding spatial bins to the Bag of Features histograms and that of cascade classifiers to improve the obtained segmentation. Our method is compared to the state of the art in the difficult Graz-02 and PASCAL 2007 Segmentation Challenge datasets.
\end{abstract}

\section{Introduction}

Currently, some of the most successful approaches to object categorization are based in bags of features $(\mathrm{BoF})$ [8]. In spite of its simplicity, this type of methods have demonstrated very good performance in many state of the art object categorization datasets [4, 19], and in other tasks such as place recognition $[3,21]$ or texture classification $[25,11]$. However, since the distribution of the local descriptors is lost in the accumulation step, it is impossible to localize the object of interest in the image and the output of the classifier is restricted to a binary present/not present label.

The most straightforward solution to overcome this limitation is to use sliding windows [24, 23], which is a generalist strategy that has no pre-assumptions on the underlying data and does not depend on the performance of a given segmentation algorithm. However, in order to perform an exhaustive sampling of the window space, millions or even billions of possible windows have to be considered, which turns the problem intractable from a computational point of view. A solution to the window search problem, with only some restrictions on the histogram normalization, was found by Lampert et al. [9], who proposed to use a branch and bound schema over the window space to direct the search and dramatically reduce the computational effort to find the optimal sub-window. Another drawback of the sliding windows approach is that only an approximate localization can be obtained, and there is no guarantee that the best classified window will contain the totality of the object. As an alternative to the sliding windows, in this work we advocate for pixel-level labeling $[10,6]$ to accurately segment class-consistent regions from the image. As in the case of sliding windows, this approach does not rely in the performance of a particular image segmentation algorithm, but region boundaries are naturally obtained from the object class conditional probabilities of every pixel. Although many variations of the base BoF method have been proposed, all of them consist on four basic steps. Namely, feature extraction from the image, feature quantization into visual words, accumulation of visual words in histograms and classification of the resulting histograms. Nevertheless, labeling every pixel of an image is a computationally demanding task, that requires carefully optimizing each step of the method if a fast implementation is required. For example, in [6], the authors propose an efficient method for each step by using a hierarchical k-means codebook [17], 
and using integral images (both for feature extraction and accumulation into histograms) with optimized small codebooks. Nevertheless, in their approach a complete integral image is necessary for each dictionary word, and the resulting histogram still needs to be classified.

Here we propose a very efficient BoF-based approach that uses the novel Integral Linear Classifier to determine the classification of an image sub-window, merging both accumulation and classification in a single step. We also contribute an evaluation of the performance and computational cost of different alternatives for dense feature extraction and codebook generation. The performance of the method is evaluated in the task of assigning a category label to each pixel of the image in the GRAZ02 dataset and the PASCAL 2007 Segmentation Challenge (VOC2007) dataset.

\section{Efficient Pixel Categorization}

A BoF approach is used to efficiently calculate the object class probabilities for every pixel ${ }^{1}$. First, densely sampled region descriptors are quantized into visual words using a codebook. Then, a fast sliding window approach is used to assign the classwise probabilities to every pixel: visual words within a window are accumulated in a histogram, which is later categorized using a linear support vector machine. Next, we show different existing alternatives to efficiently execute all the previous steps, and how accumulation and categorization can be fused into a single operation when a linear classifier is used.

\subsection{Efficient Dense Features}

Using densely sampled image descriptors as input data has several advantages when compared to keypoint-based approaches: more information can be extracted from the underlying image and the time-consuming keypoint detection step is not needed [18]. Furthermore, when image descriptors are not rotation invariant, their computation can be speeded up using integral images, which can easily make this approach even faster than the keypoint-based alternatives despite the larger number of descriptors computed. Although rotation invariance is a good descriptor property for wide baseline feature matching, Zhang et. al. [25] found that they have a negative effect in the performance of BoF approaches. In this paper we compare three different local descriptors that can be calculated using integral images: Speeded-Up Robust Features (SURF), Integral Histograms of Oriented Gradients (IHOG) and Integral Shape Context (ISC). These local descriptors are computed on image gradient values. We use Haar wavelets to calculate the image

\footnotetext{
${ }^{1}$ The source code of the proposed method can be found at http://www.cvc.uab.cat/ aldavert/plor/
}

gradient because they are less sensitive to noise [1] and can be efficiently computed regardless the scale of the feature.

\subsubsection{SURF}

The SURF descriptor [1] is obtained by accumulating the sum of Haar wavelet responses at different spatial bins. Let $d_{x}$ be the Haar wavelet response in the horizontal direction and $d_{y}$ the Haar wavelet response in the vertical direction. Then, the SURF descriptor, with $P \times Q$ spatial bins, is computed by adding the wavelets responses $d_{x}$ and $d_{y}$ over each bin sub-region. To take into account information about the polarity of the intensity images, the sum of absolute values of the responses, $\left\|d_{x}\right\|$ and $\left\|d_{y}\right\|$ is also extracted. Hence, each spatial bin of the descriptor has a four-dimensional descriptor vector with the form $\left(\sum d_{x}, \sum\left\|d_{x}\right\|, \sum d_{y}, \sum\left\|d_{y}\right\|\right)$. Finally, the resulting $4 \times P \times Q$ dimensions descriptor is L1-normalized.

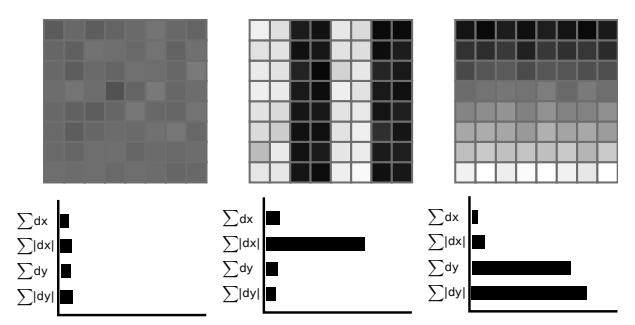

Figure 1. SURF descriptors for different image patches.

In our implementation of the SURF descriptor, the Gaussian weighting mask with the origin at the feature center is not used, so that, it can be directly computed from integral images. Thus, only $4 \times(P+1) \times(Q+1)$ memory accesses and $4 \times P \times Q$ additions are necessary to compute the descriptor, regardless of the feature region size.

\subsubsection{Integral Histogram of Oriented Gradients}

The Integral Histograms of Oriented Gradients (IHOG) [26] is an approximation of the popular SIFT descriptor [14] that can be efficiently computed with integral images. An IHOG descriptor with $N$ orientation bins and $P \times Q$ position bins, i.e. a $N \times P \times Q$ dimensional descriptor, is calculated as follows: First, $N$ orientation images, one for each orientation bin of the descriptor, are created interpolating the gradient orientation weighted by its module for each pixel of the image. Then, creating an integral image from these images, the value of an orientation bin can be calculated with only 4 additions. Therefore, only $N \times(P+1) \times(Q+1)$ memory accesses and $N \times P \times Q$ additions are needed for every IHOG descriptor, regardless of the feature size. Fig. 2 shows an example of the orientation and integral images used to calculate the IHOG descriptor.

Unlike the SIFT descriptor, the IHOG descriptor is incompatible with the Gaussian mask and the tri-linear inter- 


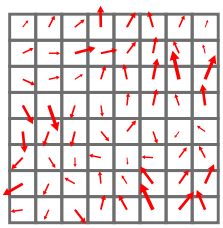

a)

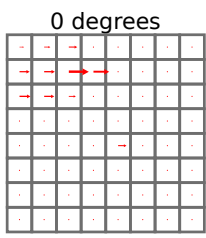

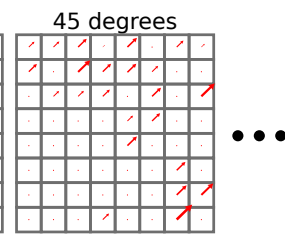

b)

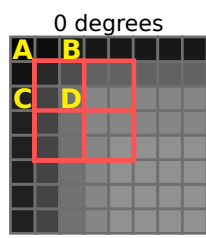

Figure 2. Example of IHOG computation: 8 orientation images b), each one corresponding to an orientation bin of the descriptor, are created interpolating the image gradient a). Then, integral images c), which are created from b), allow to compute very efficiently the IHOG descriptor. For example, the first descriptor dimension amounts to $D_{0}=D-B-C+A$, independently of the region size.

polation to weight the contribution of the gradient module in the spatial bins of the descriptor. Another difference is that the IHOG descriptor uses L1-norm instead of the L2norm.

\subsubsection{Integral Shape Context}

The shape-context descriptor [2] is obtained by accumulating gradient module in log-polar distributed bins. However, when spatial bins are distributed over a rectangular grid the descriptor can be calculated using integral images. Then, the Integral Shape Context (ISC) descriptor is computed by accumulating the module of the gradient into $P \times Q$ position bins and L1-normalizing the obtained descriptor. This descriptor can be efficiently computed using an integral image created from the gradient module. In Fig. 3, an example of how the ISC descriptor is generated from the integral of the gradient module image is shown.

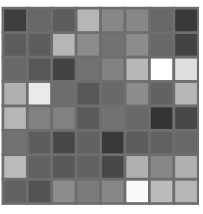

a)

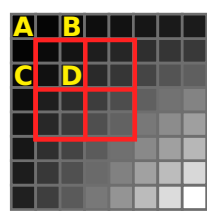

b)
Figure 3. ISC example: The integral image b) is generated from the module of the gradient image a). Then, the ISC descriptor can be calculated from the integral image b). For example, the first dimension of the descriptor is calculated as $D_{0}=D+B+C-A$.

Then, the ISC descriptor of a $P \times Q$ position bins (i.e. a $P \times Q$ dimensional descriptor) can be calculated with only $(P+1) \times(Q+1)$ memory accesses and $P \times Q$ additions, regardless of the feature regions size.

\subsection{Codebook Generation}

The computational cost of quantizing a $D$-dimensional descriptor with a linear codebook of $V$ visual words is $O(D V)$ and, since a sub-linear complexity is desirable for large codebooks, various alternatives have been recently proposed to reduce it. We evaluate two of them: the Hierarchical K-Means (HKM) [17] and the Extremely Randomized Forest (ERF) [16].
The HKM [17] defines a hierarchical quantization of the feature space. Instead of $k$ being the final number of visual words of the codebook, it determines the branch factor (i.e. the number of descendants of each node) of a tree. An HKM is generated as follows: First, the $k$-means algorithm is used to split the training data into $k$ clusters. Then, this clustering process is recursively applied to the cluster from the previous level until a maximum depth is reached or until the amount of data samples in the current cluster is lower than a given threshold. This recursive method creates a codebook with a reduced computational cost both in the training and descriptor quantization phases. The computational complexity of quantizing a $D$ dimensional descriptor using a HKM with $V$ visual words is $O\left(D k \log _{k} V\right)$. In the original implementation of the HKM, all nodes of the tree are used as visual words to alleviate misclassification problems in the superior levels of the tree and the contribution of each node of the histogram is weighted by $w_{i}=\ln \left(\frac{N}{n_{i}}\right)$, where $n_{i}$ is the number of BoF histograms that contains the visual word $i$, and $N$ is the total number of BoF histograms. However, the use of these two refinements have a modest impact in the performance of the HKM. Therefore, they are removed from our implementation.

The ERF [16] uses a combination of several random K$\mathrm{D}$ trees in order to quantize the feature space. Each K-D tree of the ERF is built recursively in a top-down manner as follows: Given a set of labeled training descriptors (i.e. descriptors with a category label associated), the K-D tree splits the training descriptors from the previous level into two disjoint sets with a Boolean test in a random bin $D_{t}$ and a random threshold $\theta_{t}$. For each split, parameters $\left(\theta_{t}, D_{t}\right)$ are generated randomly until a pair that attains an entropy value larger than $S_{\min }$ is found, or the maximum number of trials $T_{\max }$ has been reached. The parameter $S_{\min }$ can be adjusted to select the desired randomness of the K-D trees. For instance, $S_{\text {min }}=1$ creates a highly discriminant tree while $S_{\min }=0$ creates a completely random tree. The main advantage of the random K-D tree compared to other quantization methods is its low computational cost. Quantizing a $D$-dimensional descriptor vector using a random K-D tree with $V$ visual words is $O\left(\log _{2} V\right)$. Since a random K-D tree usually has less discriminative power than 
other clustering methods, several K-D trees are combined together to obtain a more discriminative codebook. Finally, the resulting $\mathrm{BoF}$ histogram is created by concatenating the histograms generated by each K-D tree of the forest.

\subsection{Integral Linear Classifier}

The integral image representation has been first introduced by Viola and Jones in [24] to quickly extract Haarwavelet type features. Since then, integral images have been applied to many different tasks like local region descriptors [26], invariant feature extraction [1] or to compute BoF histograms [6]. Inspired by these previous works, we propose to use of integral images to quickly calculate the output score of the linear classifier applied to BoF histograms. If a linear classifier is used to determine the class

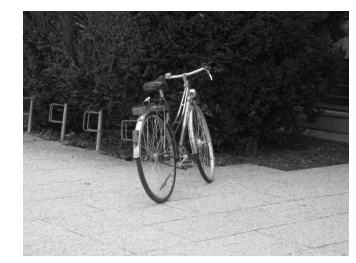

a)

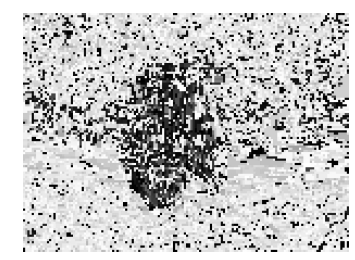

b)
Figure 4. Example of the image with the linear classifier weights for each visual words b) obtained from image a).

a $n$-dimensional BoF histogram, then the score of the linear classifier is obtained by the dot product between the $\mathrm{BoF}$ histogram $\vec{H}$ and the linear classifier weight vector $\vec{W}$, plus the linear classifier bias factor $b$ :

$$
\text { score }=\frac{1}{\|\vec{H}\|} \sum_{i=0}^{n} h_{i} w_{i}+b
$$

where $h_{i}$ is the frequency of the $i$-th visual word of the codebook, $\|\vec{H}\|$ is the norm of histogram $\vec{H}$ and $w_{i}$ is the $i$ th component of the linear classifier weight vector $\vec{W}$. If all components of $\vec{W}$ are positive, then the sum of the previous equation can be calculated using an integral image. Therefore, we define the classifier weight vector $\overrightarrow{\tilde{W}}$ components as:

$$
\tilde{w}_{i}=w_{i}-W_{\min }
$$

where $W_{\min }$ is the $w_{i}$ component with the lowest value. Then, replacing $\vec{W}$ by $\overrightarrow{\tilde{W}}$ in Eq. 1 the output score of the linear classifier is:

$$
\text { score }=\frac{1}{\|\vec{H}\|} \sum_{i=0}^{n} h_{i} \tilde{w}_{i}+\frac{W_{\min }}{\|\vec{H}\|} \sum_{i=0}^{n} h_{i}+b
$$

When the L1-norm is used to normalize the histogram $\vec{H}$, Eq. 3 can be simplified to:

$$
\text { score }=\frac{1}{N} \sum_{i=0}^{n} h_{i} \tilde{w}_{i}+W_{\text {min }}+b
$$

where $N$ is the L1-norm of histogram $\vec{H}$.

Now, an integral image can be used to calculate the sum in Eq. 4: for a linear classifier $c$, let $L_{c}(x, y)$ be the sum of components $\tilde{w}_{i}^{c}$ corresponding to the visual words at pixel coordinates $(x, y)$. In Fig. 4 an example of the $L_{c}$ image generated from the bike classifier is shown. Then, the integral image $I_{c}$ can be generated from image $L_{c}$, so that, the sum of Eq. 4 of a rectangular image region $R$ can be calculated using the integral image $I_{c}$ :

$$
H_{R}=I_{c}\left(x_{u}, y_{u}\right)+I_{c}\left(x_{b}, y_{b}\right)-I_{c}\left(x_{u}, y_{b}\right)-I_{c}\left(x_{b}, y_{u}\right)
$$

where $\left(x_{u}, y_{u}\right)$ and $\left(x_{b}, y_{b}\right)$ are respectively the upper left and bottom right corner coordinates of region $R$. Then, the output score of a linear classifier applied to any rectangular image region can be calculated as follows:

$$
\text { score }=\frac{1}{N} H_{R}+W_{\min }+b
$$

Using integral images, the computational complexity of classifying any rectangular image region is reduced to 8 memory access, 10 additions and 1 product, independently of the size of rectangular region. Furthermore, when densely sampled features are used, this computational complexity is reduced to 4 memory access, 6 additions and 1 product, as $N$ is known a priori.

\subsection{Bag of Features Spatial Bins}

Lazebnik et al. show in [12] that adding spatial information using spatial bins in the BoF histograms increases the overall performance of the method. Using spatial bins amounts to computing an independent BoF histogram for every spatial bin and concatenating them to produce the final histogram. In this case, the weights $\tilde{w}_{i}^{c}$ of the linear classifier $c$ cannot be accumulated into the same integral image $I_{c}$, but an integral image has to be calculated for each spatial bin. Therefore, the output score of Eq. 6 when $n$ spatial bins are used is:

$$
\text { score }=\sum_{i=0}^{n} \frac{1}{N_{i}} H_{R_{i}}+W_{\min }+b
$$

where $N_{i}$ and $H_{R_{i}}$ are respectively the amount of features and the integral image output in $i$-th spatial bin. The computational complexity of classifying any rectangular image region using $P \times Q$ spatial bins becomes $8 \times P \times Q$ memory access, $8 \times P \times Q+2$ additions and 1 products, independently of the size of rectangular region. Since we use densely sampled features, the computational complexity can be reduced to $4 \times P \times Q$ memory access, $4 \times P \times Q+2$ additions and 1 product, as the amount of features $N_{i}$ at each bin of the rectangular region is known a priori. 

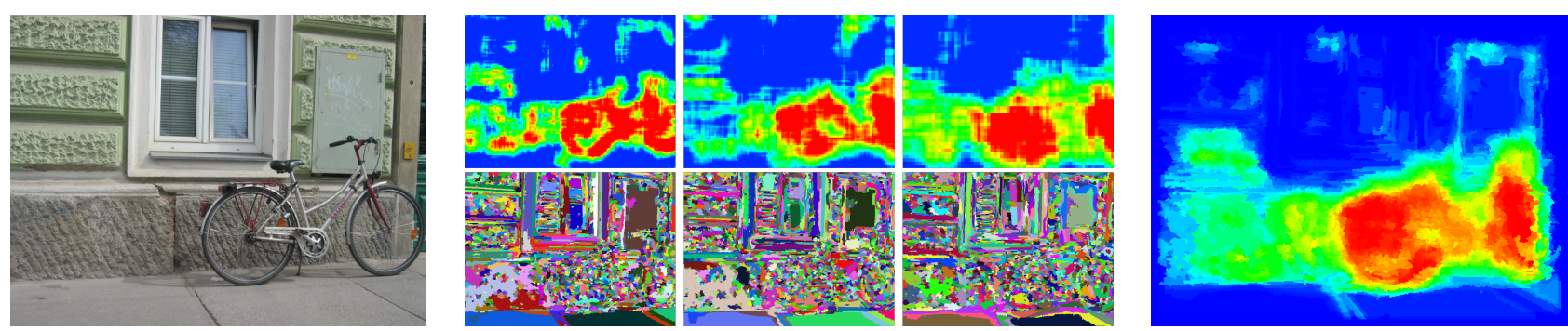

Figure 5. Segmentation example: The input image is segmented and classified at different scales (middle). Then, object segments obtained at different scales are combined in the right image, where more red represent higher probability of object. (Best viewed in color).

\section{Pixel level object segmentation}

Next we review some of the most relevant contributions to object level pixel segmentation existing in the recent literature. Fulkerson et al. [6] propose a pixel-labeling approach where integral images are effectively used to speed-up the computation of the BoF histograms without relying in suboptimal small dictionaries. Instead, in order to preserve the discriminative power of the codebook while diminishing its size, they define a coarse-to-fine-to-coarse architecture using Agglomerative Information Bottleneck to reduce a fullgrown vocabulary tree. In [7] the previous schema is reformulated to use $\mathrm{BoF}$ histograms constructed aggregating dense descriptors over a region defined by superpixel neighborhoods instead of square windows. Later a Conditional Random Field (CRF) is used to increase the precision of the method at object boundaries. Although the new schema improves the results, it is not possible to accelerate the accumulation of features in histograms by means of Integral Images as the considered regions are not square, which increases significantly the computational cost of the method.

Marszałek et al. [15] proposed the use of object masks learned from training data to accumulate the evidence provided by visual words computed from sparse local features in consistent object hypothesis, which are subsequently filtered using a discriminative classifier. Shotton et al. [22] use boosting with random feature selection to build a strong classifier using simple texton features and shape masks. A $\mathrm{CRF}$ is then used to integrate the different cues (textureshape, edge, color and location information) and object regions are finally obtained using graph cuts. Pantofaru et al. [20] proposed to combine the output of multiple image segmentation methods to overcome the limitations found by each particular approach. Pixels common to all segmented regions are grouped together assigned a class label that maximizes the marginal probability of each class in the regions that originated the common region. In this work we use this region fusion approach to integrate the results found at different scales as will be seen in next section.

\subsection{Object Segmentation}

We use the proposed Integral Linear Classifier (ILC) to efficiently assign a category label to each pixel of the image: First, integral features are densely sampled and quantized using a codebook. Then, the integral image of the linear classifier is created from the quantized features. Finally, we calculate the score of the linear classifier for all pixels of the image.

To refine the obtained object segmentation at the boundaries of the objects, the mean-shift is used to extract image regions that have an homogeneous color: we perform meanshift on a five-dimensional vector composed by LUV color space representation of each pixel and its location in the image. The mean-shift operator is applied using a uniform distribution with a window size proportional to the feature size. Then, the linear classifier score of each pixel of the image is interpreted as $P\left(c_{x, y}=k \mid I\right)=\varsigma(S(x, y))$, where $c_{x, y}$ stands for the class assigned to the pixel $(x, y), I$ is the image data, $S_{k}(x, y)$ is the classifier score for class $k$, and finally $\varsigma(\cdot)$ is the sigmoid function that remaps the output of the classifier to the $[0,1]$ interval. These probabilities are accumulated over the regions resulting from the mean-shift segmentation, so that, each region has a certain probability of belonging to an object category. Finally, instead of using this procedure in a single scale as in [6], we repeat this object segmentation procedure at different scales. To combine the resulting segmentations, we use the method proposed by Pantofaru et. al. in [20]: Pixels which are grouped together by every segmentation should be classified consistently. Thus, the "basic units" of next steps are Intersections of Regions (IofRs). Then, each IofRs is classified by combining the information from all of the individual segments that generated it. Let $i$ be an IofR, and $r_{i}^{s}$ the region which contains $i$ in segmentation $s$. Let $c_{i}$ be the class label of $i, k$ a specific class label, and $I$ the image data. Then, the segmentation integration method is:

$$
P\left(c_{i}=k \mid I\right) \propto \sum_{s} P\left(c_{i}^{s}=k \mid r_{i}^{s}, I\right)
$$

This average over the individual regions' confidence amounts to marginalizing over the regions containing $i$, as- 


\begin{tabular}{|c|c|c|c|c|c|c|c|c|c|c|c|c|c|}
\hline \multirow{3}{*}{ 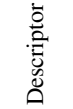 } & \multirow{3}{*}{$\begin{array}{l}\frac{y}{0} \\
8 \\
\frac{0}{0} \\
0\end{array}$} & \multicolumn{6}{|c|}{ BoF without spatial bins } & \multicolumn{6}{|c|}{ BoF with bins } \\
\hline & & & Linear & & & Cascade & & & Linear & & & Cascade & \\
\hline & & Bikes & Cars & Person & Bikes & Cars & Person & Bikes & Cars & Person & Bikes & Cars & Person \\
\hline SURF & HKM & $61.9 \%$ & $58.6 \%$ & $51.8 \%$ & $64.5 \%$ & $61.7 \%$ & $57.3 \%$ & $62.9 \%$ & $58.5 \%$ & $49.8 \%$ & $65.1 \%$ & $61.2 \%$ & $58.0 \%$ \\
\hline IHOG & HKM & $68.1 \%$ & $54.9 \%$ & $51.5 \%$ & $69.9 \%$ & $57.9 \%$ & $\mathbf{5 8 . 1 \%}$ & $68.5 \%$ & $54.9 \%$ & $48.6 \%$ & $70.8 \%$ & $58.1 \%$ & $58.6 \%$ \\
\hline ISC & HKM & $51.9 \%$ & $41.2 \%$ & $48.1 \%$ & $53.9 \%$ & $46.6 \%$ & $55.2 \%$ & $54.9 \%$ & $45.5 \%$ & $47.8 \%$ & $55.0 \%$ & $46.7 \%$ & $56.0 \%$ \\
\hline SURF & ERF & $64.0 \%$ & $61.4 \%$ & $50.9 \%$ & $65.8 \%$ & $62.6 \%$ & $56.3 \%$ & $63.5 \%$ & $61.3 \%$ & $51.0 \%$ & $66.1 \%$ & $62.9 \%$ & $57.3 \%$ \\
\hline IHOG & ERF & $70.1 \%$ & $57.1 \%$ & $50.7 \%$ & $71.3 \%$ & $58.4 \%$ & $57.3 \%$ & $69.6 \%$ & $56.1 \%$ & $51.6 \%$ & $71.9 \%$ & $58.3 \%$ & $57.2 \%$ \\
\hline ISC & ERF & $55.5 \%$ & $46.1 \%$ & $49.7 \%$ & $56.8 \%$ & $47.6 \%$ & $54.7 \%$ & $55.8 \%$ & $46.0 \%$ & $49.1 \%$ & $57.5 \%$ & $47.5 \%$ & $55.0 \%$ \\
\hline
\end{tabular}

Table 1. Precision-Recall values at equal error rate obtained in the GRAZ02 dataset for each parameter combination.

suming they are each equally probable. Finally, the class assigned to an IofR is $\arg \max _{k} P\left(c_{i}=k \mid I\right)$.

\subsection{Cascade classifier}

Unfortunately, the amount of positive training data corresponding to pixels of object categories is notably lower than its negative counterpart (usually the background samples correspond to a $90 \%$ or more of the training set pixels). In consequence, we can safely allow the trained classifier to produce up to $5 \%$ of false negatives, but this same percentage for false positives would have a disastrous effect on the precision of the method, and a much lower value (e.g. $0.01 \%$ ) is required. Given that it is very difficult to train a single classifier satisfying both conditions, we propose to deal with this asymmetry by using a cascade of linear classifiers [24]. The cascade classifier is trained using a linear support vector machine where the positive samples have double weight with respect to the background samples to guarantee that at each level of the cascade a minimum number of correct object pixels are discarded as background. To use the integral linear classifier with the cascade classifier, an integral image must be created for each linear classifier of the cascade. Then, to categorize a pixel, the different classifiers of the cascade are applied consecutively, assigning the background label if the sample is rejected by any classifier of the cascade and the object category label otherwise.

\section{Experiments}

We evaluate the performance of our proposed algorithm on two challenging datasets: the GRAZ02 [19] and VOC2007 [4]. The GRAZ02 dataset contains three object categories (bikes, cars and people) with high intra-class variation. We have used the same setup as in $[15,6]$ : The odd numbered images are taken as training and the even numbered as test. Then, the performance of the method is evaluated using pixel-based precision-recall curves. Active pixels of the ground truth segmentation mask correctly categorized as object are counted as true positives, and as false negatives otherwise. Also, incorrectly classified background pixels of the ground truth segmentation mask are counted as false positives. The VOC2007 dataset contains 21 categories with few training examples and extreme vari- ation in deformation, scale, illumination, pose, and occlusion. To train our method, we use both training and validation images of the segmentation dataset. The performance measure for the dataset is the average pixel accuracy: For each category, the number of correctly classified pixels divided by the ground truth labeled pixels. The computation times mentioned in the results have been obtained using a laptop with an Intel 2.6Ghz P9600 Core Duo CPU and 4Gb of RAM.

\subsection{Parameter Setup}

The results were obtained using the same parameters in all experiments. Dense features were extracted from square regions with sizes 20,28, 40, 56, 78 and 108 pixels, and gradient was approximated using Haar wavelets of 0.2 times the considered window size. For SURF and IHOG descriptors, we used $2 \times 2$ spatial bins, resulting in 16 and 32 dimensional descriptors respectively. For ISC descriptor, we used $4 \times 4$ spatial bins resulting in a 16 dimensional descriptor. The HKM branch factor is set to 10 , while for the ERF we use 10 trees per forest with a randomness factor set to 0.5 . Then, BoF histograms were computed accumulating the visual words inside a region which doubles the scale of the feature. Later, those histograms were categorized using a logistic regression (LR-SVM) support vector machine [13]. The SVM was trained using all BoF histograms of the training set, so that, we use a modified version of the LIBLINEAR software package [5] that takes advantage of the high degree of redundancy in BoF histograms to store them very efficiently in memory.

\subsection{GRAZ02 results}

For the GRAZ02 dataset, A codebook with 200,000 visual words in average was obtained for the HKM, while the codebook size of the obtained ERF was of 500,000 visual words in average. The cascade classifiers had 2-3 linear classifiers on average for all object categories. In Table 1 the precision-recall values obtained at equal error rate for the different configurations of the method are shown. Spatial bins in the BoF histogram had little effect in the performance of the method. However, the cascade classifier together with an ERF codebook increased the performance of the method by $2,6 \%, 2,4 \%$ and $13.12 \%$ for bikes, cars 


\begin{tabular}{|c|c|c|c|c|c|c|c|c|c|c|c|c|c|c|c|c|c|c|c|c|c|c|c|c|c|}
\hline 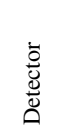 & $\begin{array}{l}\frac{y}{0} \\
8 \\
\frac{0}{0} \\
\frac{10}{0}\end{array}$ & 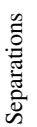 & 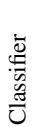 & 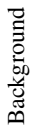 & $\begin{array}{l}\frac{8}{0} \\
\frac{\pi}{2} \\
\frac{0}{0} \\
\frac{0}{4}\end{array}$ & $\begin{array}{l}\frac{0}{0} \\
\frac{0}{0} \\
\frac{0}{0}\end{array}$ & : & 苂 & $\begin{array}{l}\stackrel{0}{E} \\
\text { E. }\end{array}$ & 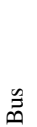 & نี & త & 胥 & ن & 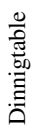 & مٌ & 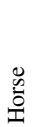 & $\begin{array}{l}\frac{9}{3} \\
\frac{0}{0} \\
\frac{0}{0} \\
\Sigma\end{array}$ & 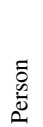 & 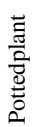 & $\begin{array}{l}\text { चे } \\
\text { जू }\end{array}$ & $\begin{array}{l}\frac{\pi}{0} \\
0 \\
0\end{array}$ & 寻 & 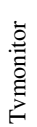 & 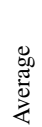 \\
\hline SURF & $\mathrm{H}$ & $\mathrm{N}$ & $\mathrm{L}$ & 49 & 12 & 7 & 9 & 10 & 8 & 35 & 15 & 19 & 3 & 12 & 17 & 12 & 13 & 19 & 38 & 15 & 12 & 10 & 18 & 23 & 17 \\
\hline IHOG & $\mathrm{H}$ & $\mathrm{N}$ & $\mathrm{L}$ & 48 & 14 & 12 & 12 & 11 & 9 & 22 & 18 & 21 & 6 & 11 & 10 & 10 & 11 & 21 & 42 & 29 & 14 & 6 & 27 & 12 & 17 \\
\hline ISC & $\mathrm{H}$ & $\mathrm{N}$ & $\mathrm{L}$ & 50 & 12 & 12 & 10 & 12 & 10 & 19 & 12 & 14 & 9 & 7 & 9 & 14 & 12 & 17 & 31 & 5 & 9 & 10 & 15 & 10 & 14 \\
\hline SURF & $\mathrm{E}$ & $\mathrm{N}$ & $\mathrm{L}$ & 50 & 14 & 12 & 9 & 13 & 12 & 18 & 20 & 13 & 25 & 19 & 19 & 17 & 8 & 34 & 25 & 24 & 14 & 11 & 25 & 28 & 20 \\
\hline IHOG & E & $\mathrm{N}$ & $\mathrm{L}$ & 47 & 11 & 12 & 38 & 17 & 8 & 20 & 24 & 28 & 21 & 19 & 15 & 7 & 10 & 21 & 16 & 5 & 24 & 11 & 38 & 22 & 20 \\
\hline ISC & E & $\mathrm{N}$ & $\mathrm{L}$ & 45 & 6 & 31 & 11 & 12 & 7 & 8 & 4 & 10 & 11 & 16 & 31 & 31 & 23 & 16 & 17 & 20 & 7 & 9 & 22 & 16 & 17 \\
\hline SURF & $\mathrm{H}$ & $\mathrm{Y}$ & $\mathrm{L}$ & 49 & 13 & 6 & 11 & 12 & 6 & 16 & 15 & 23 & 5 & 8 & 19 & 12 & 11 & 26 & 36 & 5 & 11 & 7 & 22 & 24 & 16 \\
\hline IHOG & $\mathrm{H}$ & $\mathrm{Y}$ & $\mathrm{L}$ & 48 & 11 & 22 & 12 & 10 & 5 & 22 & 16 & 13 & 4 & 17 & 9 & 5 & 7 & 23 & 41 & 9 & 14 & 6 & 26 & 10 & 16 \\
\hline ISC & $\mathrm{H}$ & $\mathrm{Y}$ & $\mathrm{L}$ & 48 & 13 & 10 & 17 & 9 & 5 & 16 & 12 & 15 & 9 & 5 & 10 & 9 & 18 & 14 & 18 & 16 & 12 & 4 & 14 & 14 & 14 \\
\hline SURF & $\mathrm{E}$ & $\mathrm{Y}$ & $\mathrm{L}$ & 45 & 7 & 34 & 11 & 18 & 15 & 22 & 14 & 12 & 7 & 14 & 35 & 12 & 6 & 32 & 29 & 33 & 9 & 11 & 36 & 33 & 21 \\
\hline IHOG & $\mathrm{E}$ & $\mathrm{Y}$ & $\mathrm{L}$ & 45 & 9 & 13 & 13 & 25 & 20 & 15 & 17 & 16 & 2 & 14 & 8 & 7 & 20 & 21 & 23 & 42 & 10 & 31 & 27 & 10 & 18 \\
\hline ISC & E & $\mathrm{Y}$ & $\mathrm{L}$ & 45 & 4 & 37 & 16 & 13 & 11 & 10 & 5 & 11 & 9 & 13 & 24 & 33 & 25 & 33 & 15 & 20 & 6 & 6 & 13 & 8 & 17 \\
\hline SURF & $\mathrm{H}$ & $\mathrm{N}$ & $\mathrm{C}$ & 48 & 21 & 5 & 13 & 10 & 8 & 21 & 28 & 15 & 3 & 7 & 16 & 21 & 16 & 43 & 44 & 16 & 20 & 8 & 38 & 28 & 20 \\
\hline IHOG & $\mathrm{H}$ & $\mathrm{N}$ & $\mathrm{C}$ & 49 & 15 & 10 & 16 & 17 & 4 & 20 & 25 & 18 & 6 & 6 & 10 & 12 & 15 & 45 & 44 & 24 & 24 & 26 & 44 & 19 & 21 \\
\hline ISC & $\mathrm{H}$ & $\mathrm{N}$ & $\mathrm{C}$ & 47 & 23 & 3 & 9 & 9 & 9 & 20 & 17 & 24 & 7 & 3 & 9 & 19 & 7 & 29 & 45 & 7 & 11 & 7 & 21 & 22 & 17 \\
\hline SURF & $\mathrm{E}$ & $\mathrm{N}$ & $\mathrm{C}$ & 48 & 22 & 7 & 13 & 12 & 15 & 22 & 22 & 18 & 8 & 13 & 21 & 19 & 24 & 50 & 26 & 21 & 25 & 13 & 37 & 28 & 22 \\
\hline IHOG & $\mathrm{E}$ & $\mathrm{N}$ & $\mathrm{C}$ & 48 & 20 & 6 & 21 & 20 & 8 & 16 & 28 & 23 & 9 & 9 & 19 & 15 & 20 & 46 & 35 & 32 & 28 & 25 & 43 & 22 & 23 \\
\hline ISC & $\mathrm{E}$ & $\mathrm{N}$ & $\mathrm{C}$ & 46 & 26 & 3 & 10 & 6 & 14 & 19 & 22 & 16 & 5 & 4 & 11 & 23 & 12 & 44 & 28 & 13 & 14 & 9 & 19 & 18 & 17 \\
\hline SURF & $\mathrm{H}$ & $\mathrm{Y}$ & $\mathrm{C}$ & 49 & 22 & 5 & 8 & 9 & 8 & 22 & 26 & 18 & 2 & 4 & 19 & 19 & 16 & 52 & 42 & 12 & 21 & 5 & 39 & 25 & 20 \\
\hline IHOG & $\mathrm{H}$ & $\mathrm{Y}$ & C & 46 & 12 & 14 & 12 & 11 & 5 & 21 & 25 & 16 & 3 & 5 & 11 & 11 & 11 & 49 & 48 & 6 & 28 & 23 & 47 & 21 & 20 \\
\hline ISC & $\mathrm{H}$ & $\mathrm{Y}$ & C & 47 & 15 & 1 & 4 & 6 & 8 & 24 & 16 & 26 & 5 & 2 & 10 & 19 & 5 & 32 & 50 & 3 & 12 & 7 & 28 & 21 & 16 \\
\hline SURF & E & $\mathrm{Y}$ & $\mathrm{C}$ & 48 & 23 & 5 & 9 & 20 & 15 & 18 & 21 & 14 & 5 & 8 & 23 & 23 & 24 & 48 & 25 & 26 & 26 & 12 & 41 & 24 & 22 \\
\hline IHOG & E & $\mathrm{Y}$ & $\mathrm{C}$ & 47 & 19 & 5 & 16 & 23 & 8 & 17 & 23 & 20 & 7 & 7 & 17 & 12 & 18 & 48 & 36 & 30 & 28 & 26 & 46 & 19 & 22 \\
\hline ISC & E & $\mathrm{Y}$ & $\mathrm{C}$ & 47 & 25 & 4 & 9 & 5 & 15 & 18 & 20 & 16 & 5 & 3 & 11 & 23 & 11 & 43 & 27 & 10 & 22 & 7 & 19 & 13 & 17 \\
\hline
\end{tabular}

Table 2. Accuracy results obtained in the VOC2007 dataset. In Codebook, H stands for HKM and E stands for ERF. In Separations, Y means spatial bins, while $\mathrm{N}$ means the contrary. Finally, in Classifier, L and C stand for linear and cascade classifier respectively.

and persons, respectively. As can be seen in the results, our method outperforms [6] and [15]. Fulkerson et al. [7] obtained better results in the categories person and cars, and similar results in the category bikes. However it must be noted that our method has the lowest reported runtime in the state of the art that we are aware of (e.g. only the very efficient conditional random field inference stage in [7] has a runtime similar to our complete method).

\subsection{VOC2007 results}

On the VOC2007 dataset, we obtained codebooks of a similar size as in the previous experiments both for the HKM and ERF, but larger cascade classifiers, with 5-6 classifiers in average. In Table 2 the accuracy results obtained with the different parameter configurations are shown. As can be seen, the ISC descriptor obtained the worst performance, while SURF and IHOG had a similar accuracy results. Again, cascade classifiers together with an ERF codebook improved the global accuracy of the method and, also like in the GRAZ02 results, spatial bins had little effect in the results. Comparing with other methods in the state of the art it can be seen that we had similar results with [20] and [22], while better results are reported for the method of [7].

\subsection{Computational Cost Evaluation}

Regarding the computational cost of the proposed approach, the average time needed to construct the codebook is of 102 seconds using HKM and 250 seconds with ERF. The time required to train a linear classifier for an object category using LR-SVM is about 3 minutes for HKM and 17 minutes using ERF. When a cascade classifier is used the time needed to train increases to 6-8 minutes for the HKM and to 25-40 minutes for the ERF, depending on the number of classifiers trained in the cascade. The time required to ex-

\begin{tabular}{cccc} 
Codebook & SURF & IHOG & ISC \\
\hline HKM & $181.7 \mathrm{~ms}$ & $298.5 \mathrm{~ms}$ & $170.9 \mathrm{~ms}$ \\
ERF & $175.7 \mathrm{~ms}$ & $293.5 \mathrm{~ms}$ & $166.7 \mathrm{~ms}$
\end{tabular}

Table 3. Average time required to extract and quantize densely sampled features in the GRAZ02 dataset images.

tract and quantize all the densely sampled features from an image of the GRAZ02 dataset (fixed size of $640 \times 480$ pixels) is shown in Table 3 for each descriptor type. Once the descriptors are computed, the time needed to obtain the image segmentation is constant regardless the number of object instances found in the images or the type of descriptor used. The computation time of the classifier for the images of the GRAZ02 dataset using the full schema (i.e. cascade classifier with spatial divisions in BoF histogram) is of $164.79 \mathrm{~ms}$ with a single object classifier: $135 \mathrm{~ms}$ for image segmentation, $2.77 \mathrm{~ms}$ for pixel categorization and $27.02 \mathrm{~ms}$ to obtain single object segmentation hypotheses from object segmentations generated at different scales. Each additional object classifier increases the total time of the method by $30 \mathrm{~ms}$. Thus, the runtime for the complete method ranges from $331.49 \mathrm{~ms}$ to $463.29 \mathrm{~ms}$ depending on the type of descriptor and quantizer used. Another very efficient pixellabeling method is the one proposed in [22], where a frame rate of $8 \mathrm{fps}$ is reported. However, in order to compare their method with the one proposed here, it must be taken into ac- 
count that their time results are obtained with images from the MSRC database $(300 \times 213$ pixel images $)$, while ours are of GRAZ02 database $(640 \times 480$ pixel images $)$ : the images used in our evaluation are 4.5 times bigger, thus the difference in computation time. When evaluated in the MSRC database the time per image needed by our method to obtain the 21-class segmentation result was of $91 \mathrm{~ms}$ (11 fps).

\section{Conclusions}

In this paper we present an efficient method to obtain a multiclass pixel-level segmentation of an image in less than 0.5 seconds. Our main contribution is the introduction of the Integral Linear Classifier, which is used to bypass the accumulation step in a Bag of Features schema and directly obtain the classification score for an arbitrary sub-window of the image. In our experiments, we show that our method obtains results comparable to the state of the art in two challenging datasets but with a much lower computational cost. Besides, we have compared the performance of three efficient feature descriptors (SURF, IHOG and ISC) and two different codebooks (HKM and ERF). Results show that the SURF descriptor gives a good compromise between speed and accuracy, the IHOG obtains better results at the price of a higher computational cost, and the ISC descriptor is the fastest to compute but has modest results. Regarding the quantizers, the ERF has shown to outperform both in accuracy and speed the HKM. Finally, we have found little improvement in the use of spatial bins in the BoF, while the cascade classifier consistently improved the results by handling the asymmetry between false positives and false negatives.

\section{Acknowledgements}

This work was supported by the Spanish Ministry of Education projects TIN 2006-15308-C02-02, MIPRCV Consolider Ingenio 2010 and the grant 2009-SGR-1434 of the Generalitat de Catalunya.

\section{References}

[1] H. Bay, A. Ess, T. Tuytelaars, and L. van Gool. Surf: Speeded up robust features. CVIU, 110(3):346-359, 2008.

[2] S. Belongie, J. Malik, and J. Puzicha. Shape matching and object recognition using shape contexts. PAMI, 24(4):509522, 2002.

[3] M. Cummins and P. Newman. Accelerated appearance-only slam. In Proc. ICRA, 2008.

[4] M. Everingham, L. Van Gool, C. K. I. Williams, J. Winn, and A. Zisserman. The PASCAL Visual Object Classes Challenge 2007 (VOC2007) Results. http://www.pascalnetwork.org/challenges/VOC/voc2007/workshop/index.html.

[5] R. Fan, K. Chang, C. Hsieh, X. Wang, and C. Lin. Liblinear: A library for large linear classification. J. Machine Learning Research, 9:1871-1874, 2008.
[6] B. Fulkerson, A. Vedaldi, and S. Soatto. Localizing objects with smart dictionaries. In Proc. ECCV, 2008.

[7] B. Fulkerson, A. Vedaldi, and S. Soatto. Class segmentation and object localization with superpixel neighborhoods. In Proc. ICCV, 2009.

[8] F. Khan, J. van de Weijer, and M. Vanrell. Top-down color attention for object recognition. In Proc. ICCV, 2009.

[9] C. H. Lampert, M. B. Blaschko, and T. Hofmann. Beyond sliding windows: Object localization by efficient subwindow search. In Proc. CVPR, 2008.

[10] D. Larlus, J. Verbeek, and F. Jurie. Category level object segmentation by combining bag-of-words models with dirichlet processes and random fields. IJCV, 2010.

[11] S. Lazebnik, C. Schmid, and J. Ponce. A sparse texture representation using local affine regions. PAMI, 27(8):1265-1278, 2005.

[12] S. Lazebnik, C. Schmid, and J. Ponce. Beyond bags of features: Spatial pyramid matching for recognizing natural scene categories. In Proc. CVPR, 2006.

[13] C. Lin, R. C. Weng, and S. S. Keerthi. Trust region newton methods for large-scale logistic regression. In Proc. ICML, 2007.

[14] D. Lowe. Distinctive image features from scale-invariant keypoints. IJCV, 60(2):91-110, 2004.

[15] M. Marszalek and C. Schmid. Accurate object localization with shape masks. In Proc. CVPR, 2007.

[16] F. Moosmann, E. Nowak, and F. Jurie. Randomized clustering forests for image classification. PAMI, 30(9):1632-1646, 2008.

[17] D. Nister and H. Stewenius. Scalable recognition with a vocabulary tree. In Proc. CVPR, 2006.

[18] E. Nowak, F. Jurie, and B. Triggs. Sampling strategies for bag-of-features image classification. In Proc. ECCV, 2006.

[19] A. Opelt, A. Pinz, M. Fussenegger, and P. Auer. Generic object recognition with boosting. PAMI, 28(3):416-431, 2006.

[20] C. Pantofaru, C. Schmid, and H. Martial. Object recognition by integrating multiple image segmentations. In Proc. ECCV, 2008.

[21] A. Ramisa, A. Tapus, D. Aldavert, R. Toledo, and R. Lopez de Mantaras. Robust vision-based robot localization using combinations of local feature region detectors. $\mathrm{Au}$ tonomous Robots, 27(4):373-385, 2009.

[22] J. Shotton, M. Johnson, and R. Cipolla. Semantic texton forests for image categorization and segmentation. In Proc. CVPR, 2008.

[23] T. Tuytelaars and C. Schmid. Vector quantizing feature space with a regular lattice. In Proc. ICCV, 2007.

[24] P. Viola and M. Jones. Rapid object detection using a boosted cascade of simple features. In Proc. CVPR, 2001.

[25] J. Zhang, M. Marszalek, S. Lazebnik, and C. Schmid. Local features and kernels for classification of texture and object categories: A comprehensive study. IJCV, 73(2):213-238, 2007.

[26] Q. Zhu, M. Yeh, K. Cheng, and S. Avidan. Fast human detection using a cascade of histograms of oriented gradients. In Proc. CVPR, 2006. 